TITLE:

\title{
The influence of tempo upon the rhythmic motor control in macaque monkeys.
}

AUTHOR(S):

Konoike, Naho; Mikami, Akichika; Miyachi, Shigehiro

CITATION:

Konoike, Naho ... [et al]. The influence of tempo upon the rhythmic motor control in macaque monkeys.. Neuroscience research 2012, 74(1): 64-67

ISSUE DATE:

2012-09

URL:

http://hdl.handle.net/2433/168067

\section{RIGHT:}

(C) 2012 Elsevier Ireland Ltd and the Japan Neuroscience Society.; この 論文は著者最終稿です。内容が印刷版と異なることがありますので、 引用の際には出版社版をご確認ご利用ください。This is the Accepted Author Manuscript. Please cite only the published version. 
The influence of tempo upon the rhythmic motor control in macaque monkeys.

Naho Konoike ${ }^{1,2}$, Akichika Mikami ${ }^{3}$, Shigehiro Miyachi $^{1}$

${ }^{1}$ Cognitive Neuroscience Section, Primate Research Institute, Kyoto University

Address: Kanrin 41-2, Inuyama-city, Aichi 484-8506, Japan

${ }^{2}$ Japan Society for the Promotion of Science

Address: Sumitomo-Ichibancho FS Bldg., 8 Ichibancho, Chiyoda-ku, Tokyo 102-8472,

Japan

${ }^{3}$ Faculty of Rehabilitation, Chubu Gakuin University

Address: Kirigaoka 2-1, Seki-city, Gifu 504-0837, Japan

Author's e-mail address:

konoike@ pri.kyoto-u.ac.jp (N. Konoike)

mikami@chubu-gu.ac.jp (A. Mikami)

Corresponding author:

Dr. Shigehiro Miyachi

Cognitive Neuroscience Section 
Primate Research Institute, Kyoto University

Kanrin 41-2, Inuyama-city, Aichi 484-8506, Japan

Tel/Fax: +81(0)568-63-5559

E-mail: miyachi@pri.kyoto-u.ac.jp

Number of pages: 14. Number of figures: 2. Number of Tables: 0 . 


\section{Abstract}

We examined behavioral features of isochronous repetitive movements in two macaques.

The monkeys were required to press a button repetitively in response to external cues. If the cue-intervals were constant (isochronous) and sub-second, the reaction time was shorter than in random-interval condition. In contrast, in the supra-second isochronous conditions, the reaction time was not different from random-interval condition. The results suggest that the monkeys can acquire isochronous rhythms if the intervals are sub-second, probably depending on the automatic timing system. However, the conscious timing system for supra-second intervals is not well developed in monkeys, unlike humans.

Keywords: non-human primates; timing; rhythm; temporal processing; behavior; forelimb movement. 
Our daily activities, such as speaking, playing music, and many kinds of sports, are rich in rhythmic movements. In order to behave smoothly, component movements should be organized in a specific temporal sequence, or a rhythm, and timed appropriately. Rhythms can emerge spontaneously during learning of motor sequences, even if it is not required (Sakai et al., 2004). On the other hand, synchronization with external rhythmic cues is useful in performance and/or learning of movement sequences. For example, people find it easier to learn dance steps or play a musical instrument with a metronome sound or visual cues provided by a conductor. Especially, interactions between auditory and motor systems are important for execution of the rhythmic movements in humans (for review, see Zatorre et al., 2007).

Numerous psychophysical and functional imaging studies have investigated motor timing mechanisms in humans using finger-tapping tasks. Psychophysical studies (reviewed by Aschersleben, 2002) showed that humans can synchronize their tapping with rhythms as slow as $0.4 \sim 0.5 \mathrm{~Hz}$ (inter-stimulus interval: 2000 2500 ms). Lewis and Miall (2003) suggested that two different mechanisms of motor timing operate depending on the time scales: an automatic timing system for sub-second intervals, and more conscious timing system for supra-second intervals.

Non-human primates also can perceive time duration and control the timing of 
discrete movements (Niki and Watanabe, 1979; Sakurai et al., 2004; Oshio et al., 2006; Leon and Shadlen, 2003; Genovesio et al., 2006; Mita et al., 2009; Yumoto et al., 2011). However, until recently, it had been unknown whether if non-human primates can control continuous and repetitive movements like humans. Zarco et al. (2009) trained macaque monkeys to perform an isochronous repetitive button press task with sub-second inter-press intervals, and showed that the characteristics of monkeys' performance were equivalent to those of human subjects. However, it is still unknown whether or not the monkeys' repetitive movements depend on the two distinct mechanisms for sub-second and supra-second time intervals, that have been found in human subjects.

To address this issue, we trained two macaque monkeys (Macaca fuscata; monkey M, 7 years old, $9.5 \mathrm{~kg}$; monkey $\mathrm{S}, 6$ years old, $6.5 \mathrm{~kg}$ ) to perform a task analogous to the synchronized tapping task, in which the monkeys were required to perform the repetitive button presses guided by isochronous rhythms of sub-second to supra-second range. All experiments were approved by the ethics committee of the Primate Research Institute of Kyoto University.

During the experiment, each monkey sat in a primate chair without head restriction. A panel equipped with a push button $(20 \times 30 \mathrm{~mm})$ was placed vertically in 
front of the monkey (30 cm from the monkey's body). A hold-lever was installed below the push button at the monkey's waist level. The button could be illuminated with a green or red light-emitting diode (LED). Each trial of the task started when the monkey held the hold-lever for $0.5 \mathrm{sec}$. After a delay, during which the button was illuminated in green, the button flashed 6 times in red with a tone (50 $\mathrm{ms}$ in duration each time). The monkeys were required to press the button 6 times when it flashed in red. If monkeys could press the button within a predetermined time-limit for 6 times, a drop of fruit juice was delivered as a reward, and the trial ended. Both monkeys pressed the button with their thumbs: monkey $\mathrm{M}$ by forward-backward arm movements, monkey $\mathrm{S}$ by pronation/supination wrist movements. There were two categories of conditions in this task: isochronous (ISO) and random (RND) conditions, differing in the temporal organization. In the ISO conditions, the LED button was illuminated in green for $1 \mathrm{sec}$ at the beginning of each trial. Thereafter, the button flashed in red with a constant frequency (stimulus onset asynchrony, SOAs: 750, 1000, or $1500 \mathrm{~ms}$ for monkey M, $600,750,1200$, or $1500 \mathrm{~ms}$ for monkey S). The SOA was unchanged within a block of trials (20 correct trials), but was changed in the next block. For the ISO conditions, the monkeys were required to press the button from a time corresponding to $15 \%$ of SOA before the cue onset (pre-cue limit) until $350 \mathrm{~ms}$ after the cue onset (for monkey M) or 
$400 \mathrm{~ms}$ after the cue onset (for monkey S) (post-cue limit). If they failed to press the button within this period, the current trial was aborted with a beep sound, and the next trial started after an inter-trial interval of $1 \mathrm{sec}$. The duration of each isochronous trial ranged from 3.05 to $7.55 \mathrm{sec}$. In the RND condition, the delay before the cue presentation was random $(1-2 \mathrm{sec})$. The SOAs were chosen randomly from four possible durations $(1,1.25,1.5,1.75$ or $2 \mathrm{sec}$ for monkey $\mathrm{M}$ and $0.75,1,1.25,1.5 \mathrm{sec}$ for monkey S). For the RND condition, monkeys were not allowed to press the button before the cue onset (pre-cue limit $=0$ ). The post-cue limit for RND condition was same as that for ISO condition. The average duration of a RND trial was $7.55 \mathrm{sec}$ for monkey $\mathrm{M}$ and $5.68 \mathrm{sec}$ for monkey $\mathrm{S}$. The dominant hand of each monkey was determined as the hand that used voluntarily to press the button during the training sessions. During the data collection, the opposite hand was restrained from reaching the button or hold-lever by an acrylic barrier attached to the primate chair. The monkeys usually performed 20 blocks, including ISO and RND blocks, in each daily experimental session. All aspects of the behavioral experiment, including presentation of stimuli and delivery of reward, were under the control of a personal computer (PC9821Xa, NEC).

The monkeys were first trained to hold the lever. In the next step, they were trained to press the button once while it was illuminated in red with a tone (cue). The 
cue duration was gradually shortened from several second to $50 \mathrm{~ms}$, and the pre- and post-cue limits were set. Thereafter, number of the cue presentation (i.e., number of the button press) was gradually increased up to 6. In this initial phase of training, only one SOA (1000 ms for both monkeys) was used. After mastering this condition, other conditions were introduced. The behavioral data were collected after the correct rate exceeded $70 \%$ in each condition. It took 8 and 10 months for monkeys $\mathrm{M}$ and $\mathrm{S}$ to reach this criterion.

We collected data in a total of 90 trials for each condition from both monkeys. The first two trials in each block were excluded from the subsequent analysis, because the monkeys could not yet know which condition was in effect at the beginning of the block. To evaluate the monkey's performance, we measured the reaction time (RT: the time from the cue onset to the response onset) as the main parameter. The RTs for the first button press in each trial were excluded from the statistical analysis, because the time of the delay preceding the first cue was fixed to $1 \mathrm{sec}$ regardless of the SOA.

Figure 1A, B shows the RTs (mean of 2nd-6th presses) for the two monkeys in each condition. The distributions of reaction times of the two monkeys for ISO750 (a sub-second interval condition), ISO1500 (a supra-second interval condition) and RND conditions are shown in the figure $1 \mathrm{C}, \mathrm{D}$. For the monkey M, a one-way analysis of 
variance (ANOVA) with conditions as a main factor revealed significant main effect for condition $(F(3,1796)=62.13, p<0.001)$. Tukey's honestly significant difference (HSD) post-hoc test showed that the RTs in the ISO750 and ISO1000 conditions were significantly shorter than that in the RND condition $(p<0.001)$. In contrast, there was no difference between ISO1500 and RND conditions. For the monkey S, a one-way ANOVA with conditions as a main factor revealed a significant main effect for condition $(F(4,2240)=102.80, p<0.001)$. Tukey's HSD post-hoc test showed that the RTs in the ISO600 and ISO750 conditions, but not those in the ISO1200 and ISO1500 conditions, were significantly shorter than that of the RND condition $(p<0.001)$. In summary, the monkeys could predict the timing of following cue in the sub-second interval conditions, but not in the supra-second interval conditions. In general, the upper limit of the interval of pacing signals with which human subjects can synchronize is about 2000 to $2500 \mathrm{~ms}$ (Aschersleben, 2002). Our results indicate that, although monkeys could predict the timing of repetitive cues, the upper limit of the interval was shorter than that in humans.

Figure $2 \mathrm{~A}$ and $2 \mathrm{~B}$ show the mean RTs for the 1 st to 6 th button presses in the ISO750 condition and the RND condition in each monkey. To compare the reaction time between the ISO and RND conditions in each button press, we performed 
a paired $t$-test. For both monkeys, the RTs in the RND condition were quite constant from the 2nd to the 6th button presses. For both monkeys, the RTs for the 1st and 2nd presses were not different between the ISO750 and RND conditions. However, for the 3rd and later button presses, the RTs in the ISO750 condition were significantly shorter compared to those in the RND condition $(p<0.05)$. This finding implies that the monkeys learn the rhythm from the interval between the 1st and 2nd cues (or perhaps the 1 st and 2 nd button presses) to predict the timing of the following button presses. Note that for monkey M, the RTs in the ISO750 condition declined during a trial. This implies that this monkey's inter-press interval was shorter than the inter-cue interval. The same tendency was observed for the ISO1000 condition of the same monkey. For monkey S, the RTs in the ISO750 condition, and also those in the ISO600 condition, were rather constant from the 3rd to 6th button presses, and were significantly shorter than those of the RND condition $(p<0.05)$. Figure $2 \mathrm{C}$ and $2 \mathrm{D}$ show the mean RTs for the 1st to 6th button presses in the ISO1500 condition and the RND condition in each monkey. For both monkeys, there was no significant difference between the RTs of the ISO1500 condition and those of the RND condition, except that the RT in the 1st button press of the monkey M was longer for ISO1500.

To examine if any learning of rhythm occurs across trials, we performed 
two-way repeated measure ANOVA with the trial number and the number of button presses as main factors. A significant main effect of the trial number was observed in the ISO750 and ISO1200 for the monkey S, and the ISO 750 for the monkey M ( $<$ < 0.05). A simple linear regression analysis showed a significant, though weak, linear trend for RT across trials for ISO750 for both monkeys $(\beta=-0.10,-0.13$ for monkeys $\mathrm{S}$ and $\mathrm{M}$, $\mathrm{p}<0.05)$, and ISO1200 for monkey $\mathrm{S}(\beta=0.09, \mathrm{p}<0.05)$. The results imply that there was a slight learning effect across trials within a block for the sub-second isochronous conditions (at least for ISO750 condition). Nevertheless, as described above, both monkeys showed clear shortening of the reaction time for later button presses of each trial (presses 3-6) if the interval was not longer than 1 sec. This may implies that monkeys' rhythm control largely depend on the automatic timing system rather than the cognitive timing system. The reason for the positive $\beta$ for ISO1200 of monkey $\mathrm{S}$ is not clear: it may reflect the fatigue.

To further assess the effects of the SOA on the acquisition of rhythm, we compared the number of trials required to reach a criterion of 20 successful trials between conditions. For monkey $\mathrm{M}$, the number of trials to criterion was $21.0 \pm 0.63$ (for ISO750), $21.4 \pm 0.87$ (for ISO1000), $25.8 \pm 0.73$ (for ISO1500), and $24.2 \pm 0.20$ (for RND), respectively. One-way ANOVA showed that a significant main effect of 
condition $(F(3,16)=12.11, p<0.001)$. Tukey's HSD post-hoc test revealed that the number of trials to criterion was significantly less in the ISO750 and ISO1000 conditions than in the ISO1500 and RND conditions $(p<0.05)$. The same tendency was observed for monkey S. The number of trials to criterion was $21.2 \pm 0.58$ (for ISO600), $21.0 \pm 0.32$ (for ISO750), $21.8 \pm 0.58$ (for ISO1200), $22.6 \pm 0.66$ (for ISO1500), and $23.2 \pm 0.89$ (for RND), respectively, though the difference did not reach the statistical significance $(p>0.05)$.

These results suggest that, for isochronous repetitive movements, monkeys can acquire the rhythm, probably depending on the automatic timing system, if the intervals between movements are short $(\leq 1000 \mathrm{~ms})$. In contrast, if the intervals are long $(>1000$ $\mathrm{ms}$ ), it is difficult for monkeys to acquire the rhythms. The cognitive timing system, needed for timing of supra-second intervals, may be less effective in motor control in monkeys than in humans.

Acknowledgements

This work was supported by a Grant-in-Aid for JSPS fellows (22.5025) to NK and by Global COE program A06. 


\section{REFERENCES}

Aschersleben, G., 2002 Temporal control of movements in sensorimotor synchronization. Brain and Cognition 48:66-79

Genovesio, A., Tsujimoto, S., Wise, S.P., 2006. Neuronal activity related to elapsed time in prefrontal cortex. Journal of neurophysiology 95:3281-3285

Leon, M.I., Shadlen, M.N., 2003. Representation of time by neurons in the posterior parietal cortex of the macaque. Neuron 38:317-327

Lewis, P.A., Miall, R.C., 2003. Distinct systems for automatic and cognitively controlled time measurement: evidence from neuroimaging. Current Opinion in Neurobiology 13:250-255

Mita, A., Mushiake, H., Shima, K., Matsuzaka, Y., Tanji, J., 2009. Interval time coding by neurons in the presupplementary and supplementary motor areas. Nature Neuroscience 12: 502-507

Niki, H., Watanabe, M., 1979. Prefrontal and cingulate unit activity during timing behavior in the monkey. Brain Research 171:213-224

Oshio, K., Chiba, A., Inase, M., 2006. Delay period activity of monkey prefrontal neurones during duration-discrimination task. European Journal of Neuroscience 23:2779-2790 
Sakai, K., Hikosaka, O., Nakamura, K., 2004. Emergence of rhythm during motor learning. Trends in Cognitive Sciences 8:547-553

Sakurai, Y., Takahashi, S., Inoue, M., 2004. Stimulus duration in working memory is represented by neuronal activity in the monkey prefrontal cortex. European Journal of Neuroscience 20:1069-1080

Yumoto, N., Lu, X., Henry, T.R., Miyachi, S., Nambu, A., Fukai, T., Takada, M., 2011. A neural correlate of the processing of multi-second time intervals in primate prefrontal cortex. PLoS one 6:e19168

Zarco, W., Merchant, H., Prado, L., Mendez, J.C., 2009. Subsecond timing in primates: Comparison of interval production between human subjects and rhesus monkeys. Journal of neurophysiology 102:3191-3202

Zatorre, R.J., Chen, J.L., Penhune, V.B., 2007. When the brain plays music: auditory-motor interactions in music perception and production. Nature Reviews Neuroscience 8:547-558 
Figure legends

Figure 1.

$\mathrm{A}$ and $\mathrm{B}$ : Mean reaction time in the isochronous (ISO) and the random (RND) conditions for monkey M and monkey S, respectively. Error bars indicate the standard deviation. ${ }^{* * *} p<0.001$. C and D: Histgram of the reaction time for the ISO conditions (interval: $750 \mathrm{~ms}$ and $1500 \mathrm{~ms}$ ) and RND conditions for monkey $\mathrm{M}$ and monkey $\mathrm{S}$, respectively.

Figure 2.

2A and 2B: Mean reaction times for the 1st to 6th button presses in the isochronous (ISO) condition (interval: $750 \mathrm{~ms}$ ) and the random (RND) condition. 2C and 2D: Mean reaction times for the 1st to 6th button presses in the isochronous (ISO) condition (interval: $1500 \mathrm{~ms}$ ) and the random (RND) condition. $\mathrm{A}$ and $\mathrm{C}$ show the data from monkey M, whereas B and D show the data from monkey S. Error bars indicate the standard deviation. ${ }^{*} p<0.05$. 
A
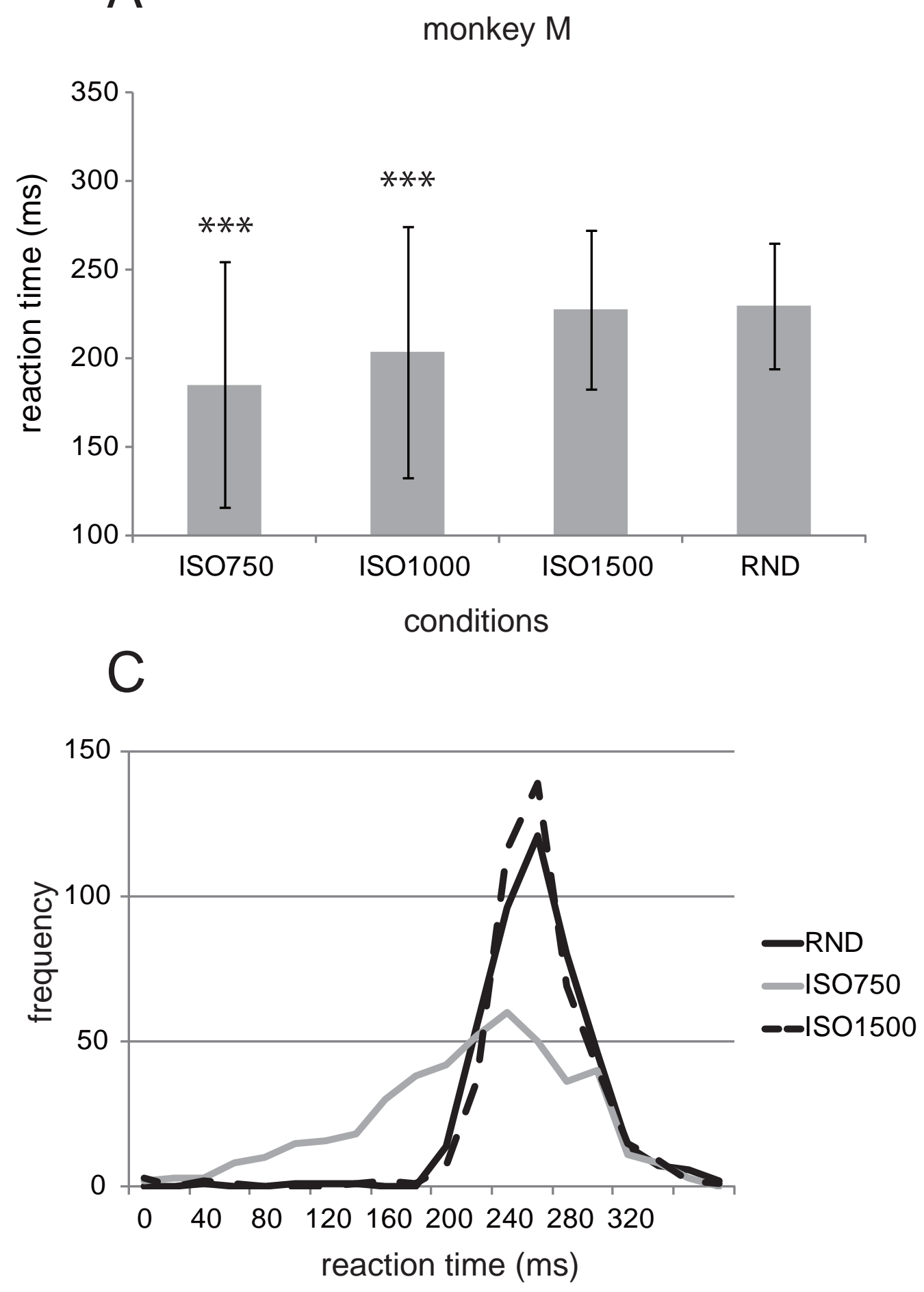

B

Figure 1.

monkey S

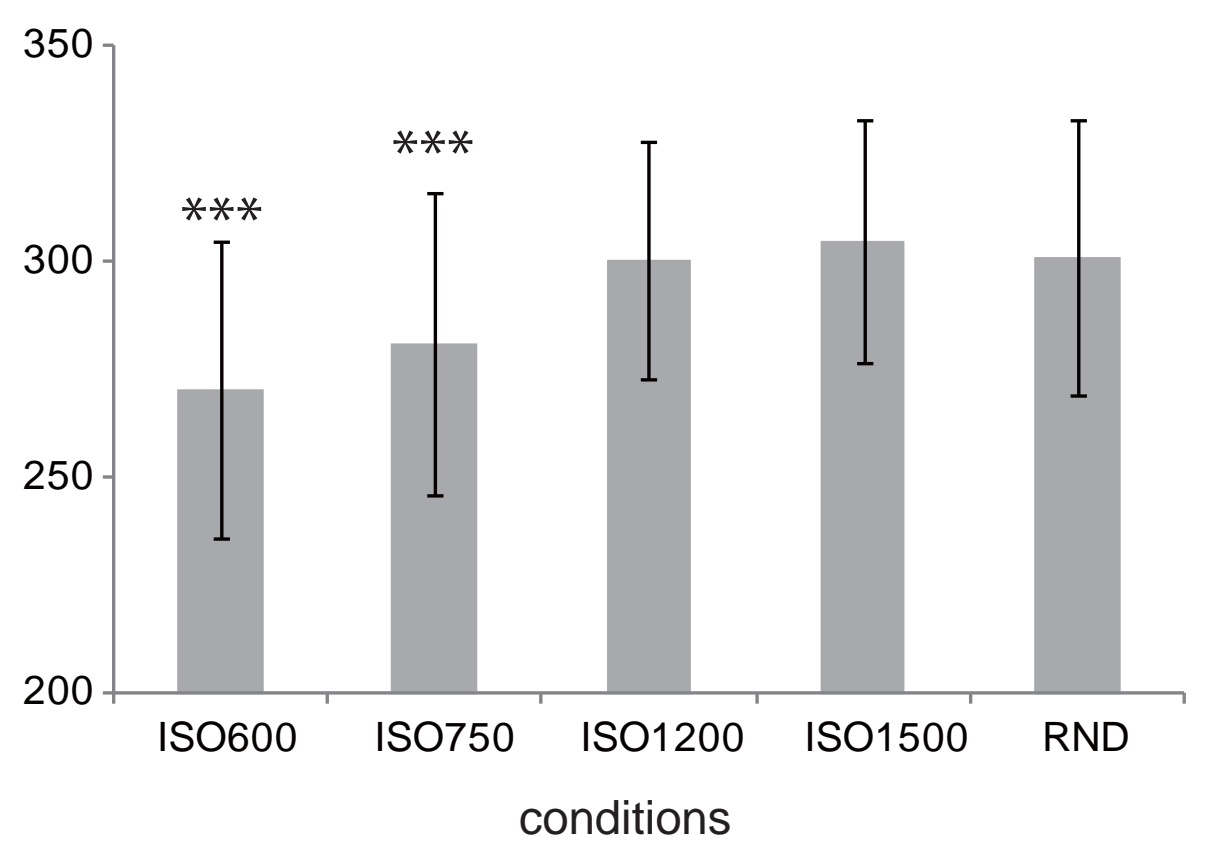

D

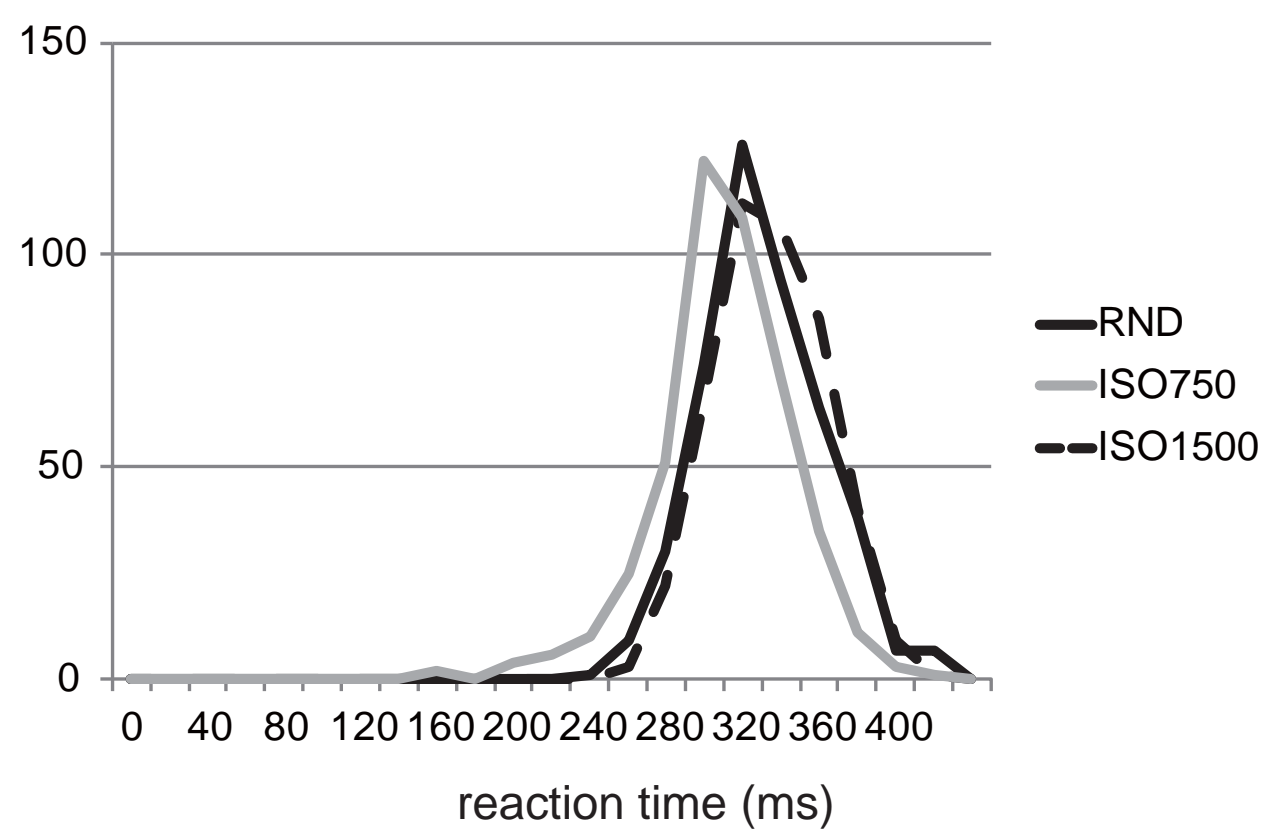


Figure 2.

A

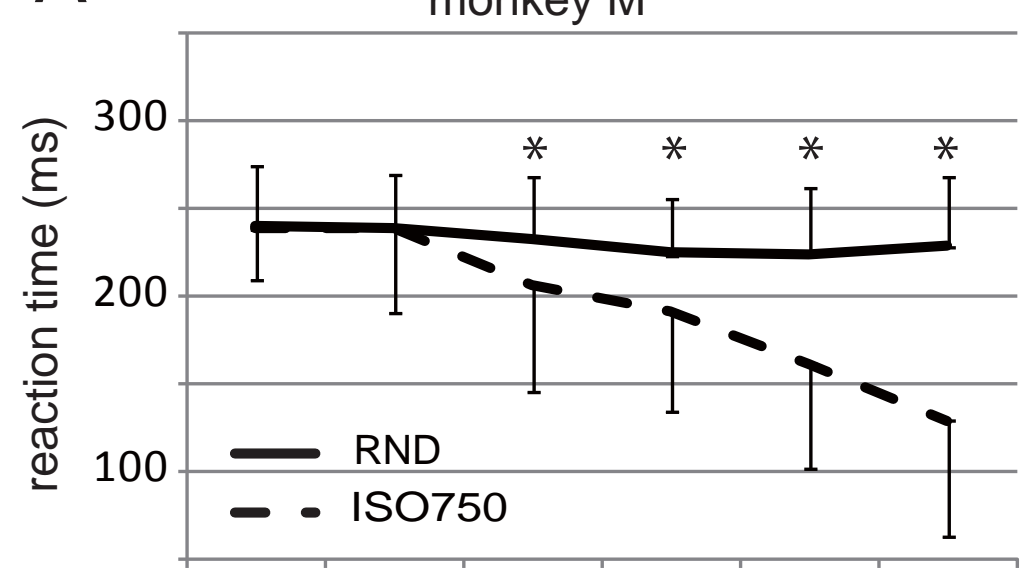

C

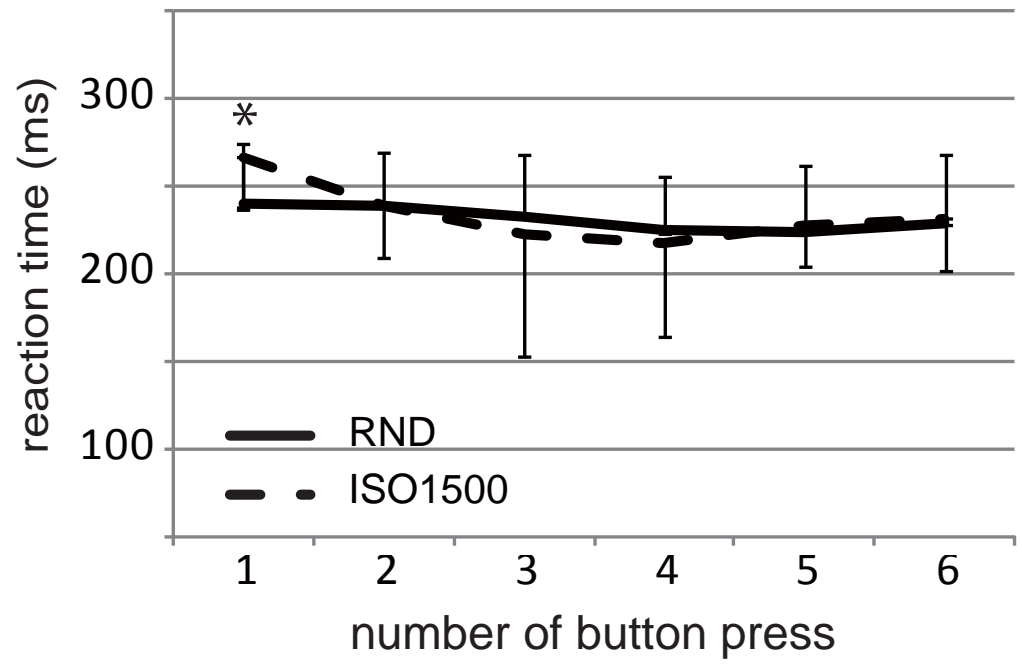

B monkey S

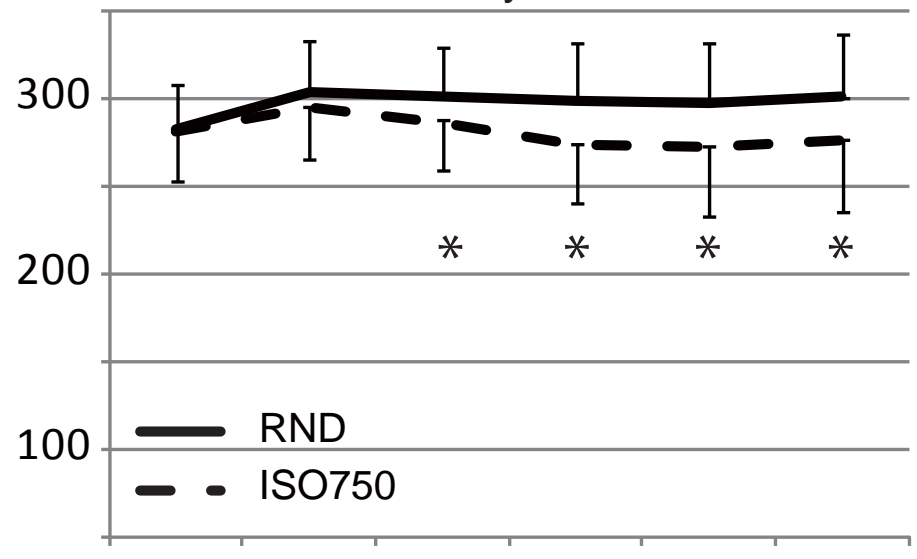

D

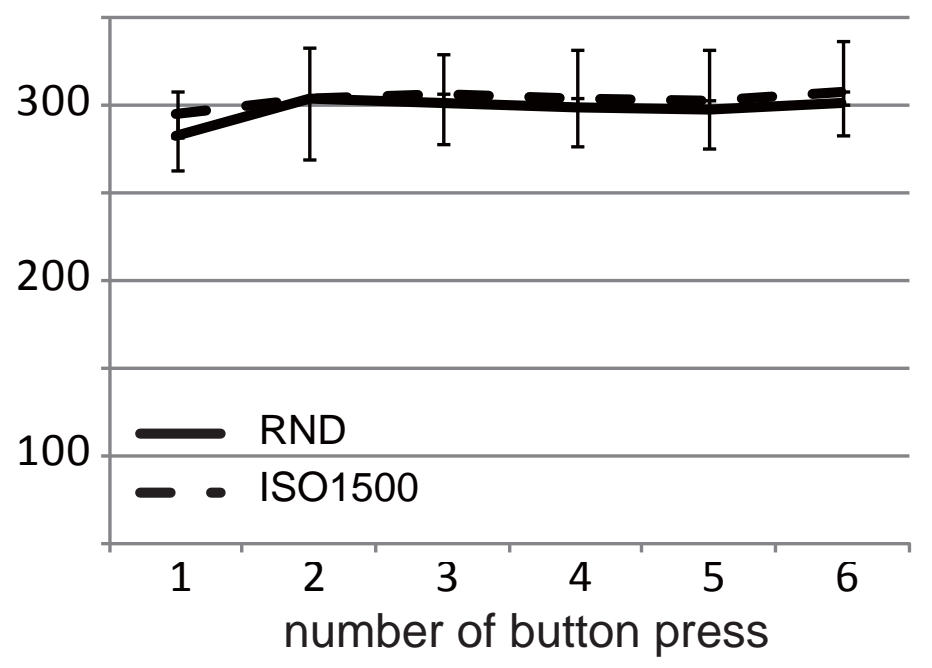

